\title{
E-Partizipation und die Grenzen der Diskursethik
}

Fritz Betz 



\title{
E-Partizipation und die Grenzen der Diskursethik
}

\section{Fritz Betz}

FH-Studiengänge Burgenland

\section{Keywords}

Demokratietheorie, Diskursethik, e-Partizipation, Leibphilosophie, Postdemokratie

\begin{abstract}
e-Partizipation benötigt Regeln für die Rechte und Pflichten der Diskursteilnehmer. Hauptsächlich werden in diesem Zusammenhang Regeln eingesetzt, wie sie ab den späten 1960er Jahren im Rahmen der Diskursethik diskutiert und später in der Theorie der deliberativen Demokratie weiterentwickelt wurden. In der Praxis der e-Partizipation finden sich allerdings Projekte mit sehr unterschiedlichen Niveaus für die Teilhabe an demokratischer Entscheidungsfindung, die oft nur bescheidene Realisierungen des in der Theorie entworfenen idealen Diskurses darstellen.

In diesem Beitrag wird zunächst argumentiert, dass mögliche Defizite der e-Partizipation bereits auf erkenntnistheoretische und anthropologische Annahmen der Diskursethik und der deliberativen Demokratie selbst zurückzuführen sind. Weiters droht e-Partizipation im politischen Kontext einer Postdemokratie zum bloßen Instrument der Akzeptanzbeschaffung für institutionelle Maßnahmen reduziert zu werden. Mit der Würdigung dieser kritischen Einwände versucht der Beitrag, Bedingungen zu skizzieren, unter denen e-Partizipation zu einer Demokratisierung beitragen kann.
\end{abstract}




\section{Inhalt}

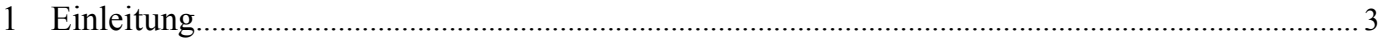

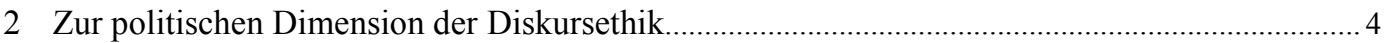

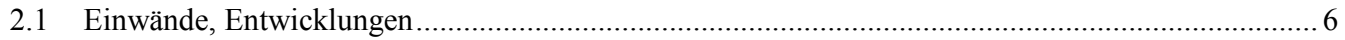

2.1.1 Diskursive Entscheidungsprobleme ……................................................................... 6

2.1.2 Leibphilosophische Einwände …………................................................................. 8

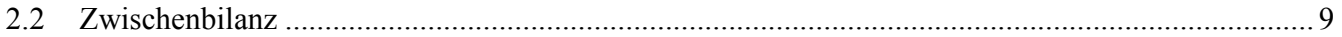

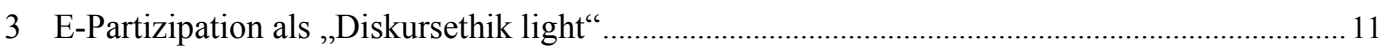

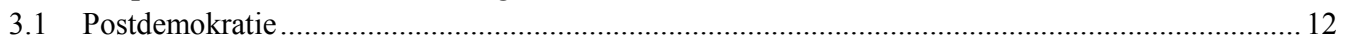

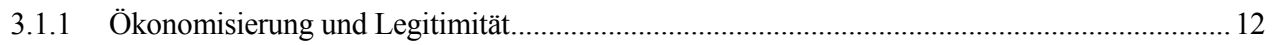

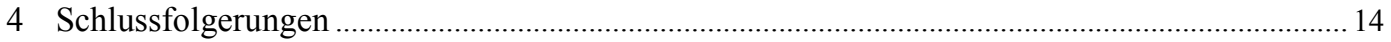

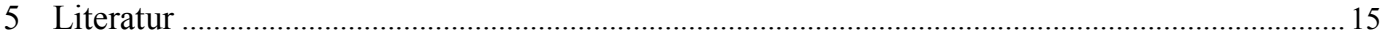

Dieser Beitrag basiert auf einem Vortrag mit gleichem Titel, den der Autor im Workshop „E-Partizipation und Klimaschutz“ der zehnten österreichischen TA-Konferenz (,,Die Ethisierung der Technik und ihre Bedeutung für die Technikfolgenabschätzung “, Österreichische Akademie der Wissenschaften, Wien, 31. 5.bis 1. 6. 2010) am 1. Juni 2010 gehalten hat.

\section{IMPRESSUM}

\section{Medieninhaber:}

Österreichische Akademie der Wissenschaften

Juristische Person öffentlichen Rechts (BGBI 569/1921 idF BGBI I |30/2003)

Dr. Ignaz Seipel-Platz 2, A-10 10 Wien

\section{Herausgeber:}

Institut für Technikfolgen-Abschätzung (ITA)

Strohgasse 45/5, A- 1030 Wien

http://www.oeaw.ac.at/ita

Die ITA-manu:scripts erscheinen unregelmäßig und dienen der Veröffentlichung

von Arbeitspapieren und Vorträgen von Institutsangehörigen und Gästen.

Die manu:scripts werden ausschließlich über das Internetportal „epub.oeaw“

der Öffentlichkeit zur Verfügung gestellt:

http://epub.oeaw.ac.at/ita/ita-manuscript

ITA-manuscript Nr.: ITA-I0-04 (Dezember/2010)

ISSN-online: | 8| 8-6556

http://epub.oeaw.ac.at/ita/ita-manuscript/ita_10_04.pdf

c 2010 ITA - Alle Rechte vorbehalten 


\section{Einleitung}

Das Begriffsverständnis von elektronischer Partizipation (e-Partizipation) ist vielfältig, und, so wird in diesem Beitrag noch argumentiert werden, diese Uneindeutigkeit kann als Vehikel politischer Instrumentalisierung genutzt werden. Weitgehend Konsens besteht zumindest darüber, dass e-Partizipation neben dem e-Voting eine der beiden wesentlichen Komponenten von elektronischer Demokratie darstellt (vgl. Macintosh 2004; Schedler, Summermatter und Schmidt 2003). Einer sehr allgemeinen Definition zufolge besteht das Wesen von e-Demokratie (und damit von e-Partizipation) in der Nutzung von Informations- und Kommunikationstechnologien (IKT) zur Unterstützung demokratischer Entscheidungsprozesse (Macintosh 2004, 1). Als anspruchsvolle Varianten der elektronischen Beteiligung gelten dabei jene, in denen Bürger als aktive Teilnehmer in elektronischer Kommunikation selbst Argumente einbringen und austauschen. Solche Praktiken werden in der theoretischen Reflexion, die es hier mit interdisziplinären Schnittpunkten von technischen und sozio-politischen Fragen zu tun bekommt, demokratietheoretisch vorwiegend dem Modell der „partizipatorischen Demokratie“ bzw. den vor allem in Kontinentaleuropa so einflussreichen Überlegungen zur „deliberativen Demokratie“ zugeordnet (siehe Lösch 2005, 166; Wilhelm 2000), wobei die Fundierung von e-Partizipation in der politischen Theorie in vielen Fällen als bemerkenswert schwach erscheint.

Ideengeschichtlich sind den Ausarbeitungen der Konturen einer deliberativen Demokratie die Entwürfe einer Diskursethik vorgelagert, also einer Ethik, die vorschlägt, die Formulierung von Verhaltensnormen nicht dem Räsonnement des vereinzelten Subjekts zu überlassen, sondern ihre Gültigkeit von der Argumentation in Kommunikationsgemeinschaften abhängig zu machen. Zunächst in diesem Zusammenhang (und erst später im Kontext der Demokratietheorie) werden jene Regeln für einen idealen, „herrschaftsfreien“ Diskurs entwickelt, auf die heute zumindest fragmentarisch und implizit rekurriert wird, wenn Diskursteilnehmer im Rahmen von Projekten der e-Partizipation Argumente austauschen.

Gleichzeitig scheint in Frage zu stehen, ob e-Partizipation das oft artikulierte Versprechen, Demokratie zu bereichern, einlösen kann. Die Schwierigkeit, in Computer vermittelter Kommunikation (CvK) Diskurse unter den Bedingungen von Gleichberechtigung und Sprachrationalität sowie mit angemessener Wirkung auf politische Entscheidungen zu organisieren, so argumentiere ich in einer theoretischen Auseinandersetzung im folgenden Abschnitt, ist bereits in den rationalistischen Prämissen der Diskursethik selbst begründet. Auf Basis dieser Prämissen wird in der Tradition des cartesianischen Subjekts zudem eine Form unvollständiger Kommunikation entworfen, aus der die Kategorie des Leiblichen ausgeklammert bleibt. In einem zweiten Abschnitt setze ich mich mit e-Partizipation im Zusammenhang mit dem von Colin Crouch (2008) geprägten Begriff der „Postdemokratie“ auseinander. Das Ziel dieser kritischen Diskussion ist es, über sie einige Anforderungen an computervermittelte Partizipation zugunsten einer Demokratisierung des Sozialen zu identifizieren. 


\section{Zur politischen Dimension der Diskursethik}

Es sind die späten 1960er und folgend die 1970er Jahre, in denen in westlichen Demokratien vehement eine weitergehende Demokratisierung eingefordert wurde. Der heute so vieldeutige Begriff der Partizipation meinte in diesem zeitgeschichtlichen Kontext nicht allein eine Stärkung des Polylogs in der Sphäre der politischen Öffentlichkeit, sondern im Kern ein Mitbestimmen und Mitentscheiden jenseits des Wahlmodus, insbesondere eine Expansion demokratischer Prozesse über die politischen Institutionen hinaus in Schulen, Hochschulen, Medienanstalten und Betriebe.

In dieser Zeitgenossenschaft lässt sich auch die Geburtsstunde der Diskursethik verorten. In einem Vortrag 1967 (in dem Sympathien mit der „Neuen Linken“ bei gleichzeitiger Absage an orthodoxe Marxismen artikuliert werden) entwirft Karl Otto Apel soziales Geschehen als eines, das in Kommunikationsgemeinschaften stattfindet, die sich aus rationalen Subjekten zusammensetzen. Dabei wird eine Ethik der Kommunikation skizziert, die sich mit der Berufung auf Vernunft gegen Monologisch-Autoritäres wendet: „Wer argumentiert, der anerkennt implizit alle möglichen Ansprüche aller Mitglieder der Kommunikationsgemeinschaft, die durch vernünftige Argumente gerechtfertigt werden können (...), und er verpflichtet sich zugleich, alle eigenen Ansprüche an Andere durch Argumente zu rechtfertigen" (Apel 1999, 424 f.).

Wer also Zustimmungsfähigkeit für seine gut begründeten Ansprüche erwartet, kann die eigene Zustimmungsfähigkeit zu den rational vorgebrachten Bedürfnissen anderer nur um den Preis eines Selbstwiderspruchs verweigern. Diese kognitivistische Modellierung einer Logik gelingender Kommunikation versteht sich zugleich als Metanorm für die Produktion allgemein gültiger Normen: Universelle Handlungsnormen lassen sich nur im Diskurs als sprachlicher Interaktion vernünftiger Subjekte gewinnen, nicht durch vereinzelte Überlegung. Apels Projekt wird dabei von zwei Anliegen getragen:

Das eine ist der binnenphilosophische, genauer, erkenntnistheoretische Versuch, die Möglichkeit einer Universalethik u. a. gegen das Argument der Relativität historischer und kultureller Kontexte, in die moralische Diskurse eingebettet sind, zu behaupten (vgl. ebenda, 362). Um es in philosophischer Pop-Prosa auf den Punkt zu bringen: Es geht um die Fortsetzung Kantischer Ethik mit einem kommunikationstheoretischen Begründungsprogramm. Der Monolog des Subjekts des Kategorischen Imperativs, eines Subjekts, dessen theoretische Befestigung in einer selbstreflexiven Moderne brüchig geworden ist, wird durch ein Apriori rationaler Kommunikation ersetzt, in Worten Apels durch die ,'intersubjektive Einheit der Interpretation' qua Sinnverständnis und qua Wahrheitskonsens“ (ebenda, 411). „Was soll ich tun?“, diese Ausgangsfrage der Kantischen Ethik wird zur Frage „Was sollen wir tun?" transformiert, die sich auch nur im Polylog beantworten lässt.

Zum zweiten aber verweist Apel, wie in einer Vorwegnahme der These von der „Risikogesellschaft“ bei Ulrich Beck (1986) oder unserer gegenwärtigen Debatten zum Klimawandel, auf die Notwendigkeit einer universalen Ethik angesichts der Auswirkungen industrieller Technik in einer von ihr hergestellten „planetarische(n) Einheitszivilisation“ (ebenda, 359, 360 f.). Dem Befund mangelt es nicht an Dramatik: Als reale Kommunikationsgemeinschaften würden wir nur überleben, wenn wir uns bemühten, ideale Kommunikationsgemeinschaften zu entwickeln (ebenda, 431). Die Vernunftmoral der Diskursethik lässt sich konzeptionell nicht von einer Ethik der politischen Partizipation trennen: Argumentationstheoretisch bezieht sie ihre Begründung durch den Rückgriff auf die pragmatischen Voraussetzungen für erfolgreiche Kommunikation in praktischen Diskursen. Als Ethik, die auf dem Prinzip des Diskurses beruht, werden durch sie wiederum universale Anforderungen an die Durchführung praktischer Diskurse, mithin ein politisch emanzipatorisches Programm formuliert. 
Im Detail werden die Anforderungen an ein solches Programm später von Jürgen Habermas präzisiert: die Offenheit des Zugangs zu Diskursen, Gleichberechtigung in gegenseitiger Anerkennung, die Offenheit des Diskurses für die Artikulation und Problematisierung aller möglichen Behauptungen, Einstellungen, Wünsche und Bedürfnisse, sowie die Notwendigkeit, zustimmungsfähige Argumente, vor allem normative, zu begründen (1983, 98f.; 1991, 132f.). Unter der Annahme, dass gelingende Kommunikation auf eine Einigung zwischen den Beteiligten hinausläuft, formulierte Habermas $(1983,103)$ seine erste Fassung eines diskursethischen Universalisierungsgrundsatzes, nach dem die Geltung von Normen der Bedingung genügen muss, dass „die Folgen und Nebenwirkungen, die sich aus einer allgemeinen Befolgung der strittigen Norm für die Befriedigung der Interessen eines jeden Einzelnen voraussichtlich ergeben, von allen zwanglos akzeptiert werden können“, bzw. das Diskursprinzip, „daß nur die Normen Geltung beanspruchen dürfen, die die Zustimmung aller Betroffenen als Teilnehmer eines praktischen Diskurses finden können (oder finden könnten).“ „Finden könnten“- der Konjunktiv ist wichtig. Ich verstehe ihn als den Konjunktiv des moralischen Abstraktionsvermögens und Induktionsverfahrens, über den der ethische Anspruch wahrheitsanaloger Gültigkeit formuliert wird, der die alltagspraktischen Prozeduren der Partizipation übersteigt: es gilt, in das reflexive Kommunikationsgeschehen die Interessen aller virtuellen Diskursteilnehmer (aller denkenden Menschen) mit einzubeziehen, auch wenn sie faktisch nicht am Diskurs teilnehmen.

Wesentlich mit Hinblick auf eine Praxis der e-Partizipation heute erscheint mir hier zunächst einmal der Hinweis, dass es in den politischen Emanzipationsbestrebungen und in den diskursethischen Debatten, wie sie vor mehr als 40 Jahren angezettelt wurden, niemals um ein wenig Teilhabe auf niederschwelligen Beteiligungsstufen ging - um mehr Transparenz, mehr Diskussion etc., sondern um die aktive Teilhabe aller mit der Fähigkeit zur Vernunftmoral Ausgestatteten an Entscheidungsgewalt im Sinn der Normsetzung. Dieser Hinweis erfüllt eine ideologiekritische Funktion, denn der Begriff der „Partizipation“ gehört (wie „Governance“, „Empowerment“ oder „e-Democracy“) heute zu den Schlüsselbegriffen im Handbuch politischer Gegenwartsrhetorik und steht unter dem Verdacht, systematische Vieldeutigkeit zu entfalten. Konkreter und angelehnt an Habermas'sche Diktion lautet dieser Verdacht: Die Appelle an „verständigungsorientierte Kommunikation“ bemänteln strategische Kommunikationsabsichten, wenn politische Institutionen e-Partizipation „top-down“ u. a. zur Technikfolgenabschätzung organisieren. „E-Partizipation soll den Bürgerinnen und Bürgern die Möglichkeit geben, sich über den Verfahrensstand in der europäischen Gesetzgebung zu informieren“ und „Entscheidungen aus Brüssel besser nachvollziehen“ zu können, sagte etwa der Abgeordnete des EU-Parlaments, Matthias Groote $(2009,6)$ in seiner Eröffnungsrede zum „eParticipation Day 2009“ der Europäischen Kommission. Während die Rede konkrete Beispiele für solche Informationsmöglichkeiten enthält, fehlen Präzisierungen für die ebenso erwähnte Vision, die Bürger ,auf allen Ebenen der demokratischen Entscheidungsfindung mit einzubeziehen“ (ebenda, 4). Die Rhetorik verweist auf angestrebte Akzeptanz- und Legitimationsgewinne, während sie offen lässt, ob und wie das Handlungsspektrum einer „schwachen“, weil weitgehend passiven politischen Öffentlichkeit erweitert werden sollte. Im Gegensatz dazu sind die Projekte zu einem diskursethischen Moralprinzip bis in die 1980er Jahre hinein radikal: sie entwerfen als Ideal die völlige Übernahme des Entscheidungsprozesses durch die Rationalität des Diskurses, damit konkret durch gleichberechtigte DiskursteilnehmerInnen. Den Horizont der umfassenden Beteiligung an Entscheidungen teilen sie mit sogenannten „sozialen“ oder „beteiligungszentrierten“ Demokratietheorien, sie übersteigen aber die Sphäre des konkret Politischen mit dem Anspruch der Universalität. 


\section{I Einwände, Entwicklungen}

Bevor ich die politische Dimension einer möglichen Instrumentalisierung von e-Partizipation weiter verfolge, möchte ich zunächst aus dem weit verzweigten Archiv der Grundsatzdebatten zur Diskursethik zwei Stränge der kritischen Argumentation skizzieren, die mir für eine Praxis von Beteiligungsverfahren in elektronischen Räumen wesentlich erscheinen. Das ist erstens die Frage nach den Grenzen der Potenziale der Diskursrationalität in der Konfliktbewältigung und Entscheidungsfindung: Sie berührt den für e-Partizipation zentralen Aspekt des Umgangs mit Konsens und Dissens. Zweitens ist das die Frage nach den anthropologischen Voraussetzungen der Diskursethik: Ihr kognitivistisches und sprachzentriertes Menschenbild legt es im Zusammenhang mit e-Partizipation nahe, die medientheoretische Debatte um die kommunikativen Möglichkeiten und Einschränkungen von CvK explizit auf den Rahmen demokratischer Willensbildung zu beziehen.

\section{I.I Diskursive Entscheidungsprobleme}

Gegen Habermas' Modell einer Konsensfähigkeit intersubjektiver Geltungsansprüche im theoretischen Unterbau der Diskursethik kann aus einer soziologischen Perspektive bestritten werden, dass diese Konsensfähigkeit bereits im Medium der Sprache selbst hinreichend verankert sei. Ich übergehe dabei die fundamentalen Gegenpositionen zur kognitivistischen Grundannahme einer strukturellen Rationalität von Sprache, wie sie von poststrukturalistischen Theoretikern vertreten werden (vgl. Zizek 1991, 1997) und konzentriere mich zunächst auf eine Argumentation, in der die sozialen Einschränkungen diskursiver Rationalität im Vordergrund stehen. So hat etwa Dorothea Jansen (2000, 187-190) in ihrer Analyse des vom Wissenschaftszentrum Berlin Anfang der 1990er Jahre ausgerichteten Gentechnikdiskurses Bestätigungen für die These gefunden, dass Wert- und Tatsachenkonflikte zwischen Diskursteilnehmern auf der Basis des „,besseren Arguments“ nicht vermittelbar seien. Bestritten wird dabei nicht, dass erst reflexiv interagierende Subjekte zu Verständigung überhaupt gelangen können, aber es wird deutlich auf die Grenzen in der gegenseitigen Anerkennung von Argumenten verwiesen.

Im Fall von Tatsachenkonflikten nimmt Jansen die konstruktivistische Perspektive ein, dass es keine standortungebundene objektive Instanz der Entscheidung geben könne (ebenda, 188). Um dieses Argument mit Hinblick auf zeitgenössische Debatten zu veranschaulichen: Die konstruktivistische Relativierung von Wahrheitsfähigkeit erscheint dem Common Sense für einfache assertorische Sätze wie „Der Vernagtferner in den Ötztaler Alpen verlor zwischen 1846 und 2003 etwa zwei Drittel seiner Masse" wenig einsichtig, sie wird aber mit zunehmender Komplexität von Sachverhalten und der Optionen ihrer Begründung und Kontextualisierung plausibler, vollends aber bei Urteilen, die (zukünftige) Möglichkeiten ausdrücken wie: „Durch die Klimaänderung wird es auch in den Tropen weniger Niederschläge geben. Die Folgen sind bereits heute schon erkennbar, Waldbrände wüten weiter, Dürre und Austrocknung der Flüsse, Lebewesen verenden qualvoll und Pflanzen verwelken“(Wetter-Klimawandel.de 2010). Abgesehen davon, dass sich diskursive Alltagspraxis nicht im Modus sprachlogischer Stringenz der verschrifteten philosophischen Reflexion realisiert: Der Möglichkeitsraum kontextgebundener, vor allem lebensweltlich geprägter Beobachtungsstandpunkte zu den eben behaupteten Sachverhalten ist nicht zu begrenzen. Weder die kommunikative Teilnahme (durch die sich standortgebundene Beobachtungen verändern können), noch die Begründungsmodi rationalen Sprachgebrauchs sind im Konfliktfall hinreichende Brückenprinzipien, um die Differenzen in der Wahrnehmung und Interpretation von Tatsachen zu tilgen.

Wertkonflikte, so wird in der Analyse des Gentechnikdiskurses argumentiert, lassen sich nur mittels „Setzung“ durch (rechtlich) autorisierte Instanzen lösen, und nicht mehr durch Logiken der Begründung. Werthaltungen sind Resultate von Sozialisierung, sind sozio-kulturell geprägt und so- 
zialstrukturell verankert (Jansen 2000, 187 f.). Eine sprachlogische Unentscheidbarkeit von Wertkonflikten wird gesellschaftspolitisch regelmäßig spürbar, wenn wir uns in Auseinandersetzungen um die Priorität von Werten befinden, wie sie im Grundrechtskanon verankert sind: so etwa in den rezenten Debatten um das Prinzip der Privatsphäre in elektronischen Räumen, wo sich die Angemessenheit freiheits- oder sicherheitsorientierter Begründungen nicht mehr argumentativ, sondern nur auf Basis diskursiver Hegemonie entscheiden lässt.

Solchen Entscheidungsproblemen trägt Habermas in seinen Untersuchungen zu Rechtsstaatlichkeit und diskurstheoretisch begründeter (deliberativer) Demokratie in den 1990er Jahren, gebündelt in „Faktizität und Geltung“ (1994), Rechnung. Unter anderem ist hier auch von „kognitiver Unbestimmtheit“ die Rede, die von der „Faktizität der Rechtsetzung absorbiert“ werden müsse (1994, 147). Das liest sich zunächst wie eine Konzession an die Kritik der kognitivistischen Untermauerung der Diskursethik. Als explizite Motive für die Abschwächung einer Erwartungshaltung gegenüber dem diskursiven Moralprinzip im Rahmen demokratischer Gemeinwesen erscheinen bei Habermas dann aber die Sorge um die private Autonomie (die es als „negative Freiheit“, also als „Freiheit von etwas“, damit auch als Freiheit von der kommunikativen Freiheit eines diskursethischen Regimes zu schützen gelte) und jene um die Unverbindlichkeit „postkonventioneller Moral“ (ebenda, 148-151), der die sozialintegrative Kraft des Rechts entgegengesetzt wird. Eine hypothetische Herrschaft des Moralprinzips wird damit einer zweifachen Kritik unterzogen. Im Hinblick auf private Autonomie wird ihr das Potenzial des Totalitären zugesprochen, mit Hinblick auf die Durchsetzung von Handlungsnormen in der politischen Öffentlichkeit mangelnde Rigorosität. Habermas hält konsequent am Leittheorem verständigungsorientierter Kommunikation fest, möchte aber die Arena der Diskursethik begrenzt wissen. Recht und Moral werden dabei als komplementäre Sphären konzipiert, wobei die Gestaltung von Rechtsnormen zwar von diskursethischen Prinzipien getragen und an eine diskursethisch agierende Öffentlichkeit gekoppelt sein soll, Entscheidungen aber (in theoretischer Replikation des formalen politischen Status quo in repräsentativen Demokratien) an rechtlich geregelte Verfahren delegiert werden (vgl. ebenda, 362-364.).

Diese Trennung zwischen moralischen Begründungsdiskursen und verfahrensregulierten Verhandlungen als „Anwendungsdiskursen“, in denen das Moralprinzip durch den „Grundsatz der Angemessenheit“" (ebenda, 140) ergänzt wird, ist problematisch. Habermas' Argumentation ist insgesamt stark verfahrensorientiert, benennt aber für die Schnittstellen von Moral und Recht keine überzeugenden Vorgangsweisen mehr, durch die ein diskursethisch inspiriertes und von Entscheidungen „entlastetes“ öffentliches Räsonnement mit den entscheidungsbefugten politischen Teilsystemen konkret verknüpft wäre, oder umgekehrt, durch die formale Verfahren rechtsstaatlicher Organe durch diskursethische Prinzipien zwingend angereichert würden. Der grundlegende Hinweis darauf, dass die Legitimität von Recht und institutionalisierter Politik von der Qualität des öffentlichen Diskurses abhängen, macht noch nicht eingängig, warum sich in der öffentlichen Sphäre verständigungsorientierte Kommunikation gegen die Machtkalküle strategischer Kommunikation durchsetzen sollte. So bleibt nur der idealistische Aufruf, die öffentliche Debatte dürfe „nicht im schwarzen Kasten einer Operationalisierung verschwinden, die sich mit grobmaschigen Indikatoren zufriedengibt“" (ebenda, 369).

Das Projekt der deliberativen Demokratie mit dem Telos von Konsens oder zumindest von Kompromissen steht des Weiteren in scharfem Widerspruch u. a. zu einer Hegemonietheorie, wie sie von Ernesto Laclau und Chantal Mouffé (1991), später vor allem von Mouffé mit den Begriffen der radikalen, pluralen oder agonalen Demokratie entwickelt wurde. Hier sind antagonistische Beziehungen ein grundlegendes Merkmal politischen Geschehens, denn erst durch sie werden kollektiv geteilte Identitäten hergestellt und Interessenslagen politischer Akteure konstruiert (Laclau und Mouffé 1991, 176 ff.). Um Wertkonflikte wie weiter oben beschrieben kann sich dann innerhalb von Demokratien eine Pluraliät von kollektiven Identiäten entfalten. Durch die gegenseitige Anerkennung als Staatsbürger werden Feinde (Antagonisten) zu Gegnern (Agonisten). Motor demo- 
kratischer Vitalität bleibt aber die konflikthafte Natur pluralistischer Verhältnisse, während zu starke Konfliktvermeidung und Konsensorientierung lähmend auf die Bereitschaft zur politischen Partizipation wirken (vgl. Mouffé 2000, 13 f.).

Ein gutes Beispiel für die Problematik starker Konsensorientierung bietet im Internet zugängliche Open-Source-Software wie Candiwi, Metascore, Vilfredo oder Votorola, als deren Entwicklungsziel unter anderem die Effizienz von elektronischen Debatten ausgewiesen wird (vgl. Candiwi 2010, Metagovernment 2010). In unterschiedlichen Varianten und Entwicklungsstadien unterstützen diese Applikationen die Identifizierung semantischer Übereinstimmung und das Ausfiltern strittiger Argumente. Anschaulich wird dabei, dass durch die Fokussierung auf Konsens jene Interessensgegensätze in den Hintergrund treten müssen, die in der politischen Moderne nicht nur toleriert, sondern in Abgrenzung zu vor- und anti-demokratischen Gesellschaftsmodellen in sozialer und institutioneller Ausdifferenzierung aktiv gepflegt werden.

Mit Jacques Derrida (2003, S. 130, 150f.) kann der prozesshafte, der „kommende“ Charakter der Demokratie betont werden (,démocratie á venir“), und damit, dass großzügige Zeitdimensionen zu ihren wichtigsten Ressourcen zählen. In einer Güterabwägung zwischen effizienten Verfahren der Entscheidungsfindung und zeitintensiver Eröffnung von Teilhabechancen bevorzugt Demokratisierung in der Moderne mit ihrem Grundzug hin zu einer Fragmentierung von Macht im Zweifelsfall letztere. Damit wird nicht nur eine Unterscheidung zu modernen Totalitarismen getroffen, die sich mitunter auch über den Begriff der Effizienz legitimieren. Auch in der funktionalen Differenzierung der Moderne zwischen Politik und Wirtschaft als unterschiedlichen Teilsystemen ist Effizienz als ökonomisches, und nicht primär politisches Leitmotiv zu verstehen.

\section{I.2 Leibphilosophische Einwände}

Für den zweiten Strang kritischer Gegenpositionen zum diskursethischen Projekt muss zunächst daran erinnert werden, dass sich Konzepte, die Antworten auf die Frage „Was soll ich tun?“ suchen, nicht von den Antworten auf eine weitere grundsätzliche Frage der Kantischen Philosophie trennen lassen, nämlich von jenen auf die Frage „Was ist der Mensch?“. Subjekt (und Objekt) der Diskursethik ist der Mensch als Vernunftwesen, das in der Tradition der cartesianischen Trennung von Körper und Geist steht. Unter solchen anthropologischen Voraussetzungen, so plädierte zuletzt Elisabeth List $(2009,15)$ in einer phänomenologisch unterlegten „Ethik des Lebendigen“, werden wir von jenen Anteilen unserer Existenz abgeschnitten, die ,,vor aller Reflexion und bewusster Intentionalität" liegen. Sinnstiftend sind wir aber nur als sinnliche, als empfindende und fühlende, als mit einem Körpergedächtnis, als mit lebendigen Erfahrungen ausgestattete Leib-Subjekte. Lebendige Erfahrung ist das Sediment für unsere kognitiven Leistungen. Leibhaftigkeit, das ist in den Worten Gernot Böhmes (2003, 63) „Natur, die wir selbst sind“, aber eben als jeweils unterschiedlich sozialisierte und kulturalisierte Natur mit der besonderen Fähigkeit, zu ihr eine reflektierte Haltung, oder mit den Begriffen Helmuth Plessners, eine „exzentrische Positionalität“ einzunehmen.

Besondere Brisanz bekommt dieser Ansatz in Lists Auseinandersetzungen mit der zeitgenössischen Bioethik, die sich als angewandte Ethik mit den Folgen technisch-medizinischer Eingriffe in elementare Lebensprozesse beschäftigt, damit auch Aussagen über die definitorischen Grenzen für menschliches Leben und über dessen „Wert“ trifft. Normadressaten und -schöpfer einer Vernunftmoral sind Personen, deren kognitive Ausstattung sie entscheidungsfähig macht. Wer oder was außerhalb dieses Zirkels steht, wird insbesondere im utilitaristischen Kalkül zum Objekt instrumenteller Rationalität degradiert. Eine solche Instrumentalisierung, so argumentiert List, findet aber nicht erst dann statt, wenn individuelles menschliches Leben zum bloßen Mittel für die Zwecke einer übergeordneten Ökonomie des Nutzens wird, sondern ist bereits in den „epistemologi- 
schen-rationalistischen Prämissen“" (List 2009, 92) angelegt, die das Leib-Subjekt einer künstlichen Trennung im Geist-Körper-Dualismus unterwerfen. Dem Hauptstrom der Bioethik sei vorzuwerfen, er dekretiere „einen moralisch höchst fragwürdigen Umgang mit dem Leben von Menschen in Grenzsituationen, deren Leben als Lebendigsein von diesen Prämissen her nicht respektiert werden muss, weil sie das Kriterium des Personseins nicht erfüllen“ (ebenda). Grundlegende Rechte im ethisch-moralischen Universum dürften, so die Konsequenz, nicht am Status als vernunftbegabter Person festgemacht werden, sondern wären mit Kriterien wie Lebendigkeit, Lebens- und Beziehungsfähigkeit zu begründen (ebenda), Kriterien, die auf vitalen Funktionen wie Kreativität, Spontaneität, Empfindungsfähigkeit, Orientierungsfähigkeit bzw. Responsivität beruhen (vgl. ebenda 15, 19, 36f., 106).

\subsection{Zwischenbilanz}

Lässt sich aus solchen Einwänden und Überlegungen etwas für Projekte der e-Partizipation gewinnen? Die generelle Problematik einer Konsensorientierung (Laclau und Mouffé) und die Grenzen der Entscheidungsfähigkeit in praktischen Diskursen (Jansen) sprechen in keiner Weise für eine Abkehr vom Apriori kommunikativer Intersubjektivität bei der Bearbeitung gesellschaftspolitischer Fragen in CvK, sondern können die Motivation bilden, gesteigerte Ansprüche an die Settings diskursiver Praktiken zu stellen. Ein kritischer Punkt für die Diskurspraxis bleibt dabei das Moment der Entscheidung über Handlungsnormen. Werden öffentlich organisierte Diskurse von ihm entkoppelt, erübrigen sie sich, weil sie die Autonomie- und Souveränitätsansprüche der Beteiligten grundlegend enttäuschen. Die politische Herausforderung liegt einerseits darin, Konzepte zur Erweiterung des Terrains entscheidungsbefugter Körperschaften zu erproben: Das könnte im Fall der e-Partizipation bedeuten, verstärkt lokalen bzw. numerisch kleinen Diskursöffentlichkeiten direktdemokratische Entscheidungsbefugnisse einzuräumen. Das Konsensprinzip wäre dabei durch Verfahren formalisierter Mehrheitsfindung zu ersetzen, die an Deliberation mit ihrem Akzent auf der Notwendigkeit von Begründungen für zustimmungsfähige Argumente anschließen. Andererseits gälte es, die Interdependenz von Urteilsbildung in einer breiten Öffentlichkeit und institutionalisierter Entscheidung durch Verfahren transparenter zu gestalten: Werden Vorschläge aus einer breiten öffentlichen Debatte an kleinere Gruppen politischer Repräsentanten oder Experten delegiert, müsste nicht nur nachvollziehbar sein, dass diese Vorschläge aufgenommen wurden, sondern auch, von wem und aus welchen Gründen diese Vorschläge in der Entscheidungsfindung unterstützt bzw. abgelehnt werden. ${ }^{1}$

Eine andere Konsequenz könnte eine Erweiterung des Diskursbegriffs und -prinzips selbst sein. Elisabeth Lists Thesen eröffnen für eine Praxis ethisch-politischer Diskurse wichtige Fragen: Wenn sie im Sinn einer leiborientierten „conditio humana“ die Rechtsansprüche von Menschen betont, die das Kriterium kognitiver Bewusstheit nicht erfüllen, so fordert das weitere Auseinandersetzungen um die Bestimmung und Ausgestaltung dieser Rechte. Das berührt auch die Frage, wer unter welchen Umständen auf welche Weise zum Normproduzenten oder zum Normadressaten bzw. beidem werden kann. Eine der logischen Folgerungen aus Lists Ausführungen wäre es, dass es der Wahrnehmung unseres Lebendig-Seins durch uns selbst bedarf, wenn wir als Schöpfer von Handlungsnormen auftreten. Dies setzt wiederum voraus, dass wir bereits die onto- und phylogenetische Leistung erbracht hätten, über ein (gefühltes) Wissen um uns als Leib-Subjekte zu verfügen. Ein

1 In Ansätzen lässt z. B. der „Beteiligungsprozess zur Klimawandelanpassungsstrategie“ des österreichischen Umweltbundesamts ein solches Modell erkennen (siehe Stickler, Prutsch und Balas 2010). 
solches Wissen muss sich mit der Evidenz von Leiblichkeit ebenso wie mit der gesellschaftlichen Unterdrückung von Leiblichkeit auseinandersetzen: Auch wenn sich unser Leib (in Schmerz- oder Lusterfahrungen) unumgänglich aufdrängt, gilt es immer wieder, ihn erst gegen kulturbedingte Selbstentfremdung und eine Ökonomie der Dressur von Wahrnehmung und Artikulation zu gewinnen. „Moralische Fragen im Bereich des Leiblichen verlangen.. ., dass man in seinem Leib wohnt, dass man vertraut mit sich ist" (Böhme 2003, 79). Eine solche Vertrautheit setzt die Übung des Wahrnehmens, Empfindens und Fühlens in dynamischer Beziehung zu einer exzentrischen Positionalität voraus, was als lebenspraktisches Programm noch um einiges anspruchsvoller erscheint als die Beschränkung auf den Vollzug kognitiver Leistungen im Gefolge eines cartesianisch geprägten Subjektbegriffs.

Wie wäre es aber, ethisch-politische Diskurse selbst als Übungsfeld eines solchen Programms aufzufassen, d. h. nicht nur, wie in den frühen diskursethischen Entwürfen formuliert, der Artikulation aller möglichen Bedürfnisse Raum zu geben (s. o.), sondern diese Diskurse mit gesteigerter Aufmerksamkeit für die Artikulationen von Empfindungen und nonverbalen Äußerungen zu versehen? Als Mindestanforderungen müsste für das Setting von Diskursen dann zumindest temporär leibliche Ko-Präsenz der Beteiligten gefordert werden, vor allem aber die leibliche Präsenz jener, die von den verhandelten Inhalten unmittelbar betroffen sind. Das bedeutet konkret: Die Medienkanäle elektronischer Kommunikation vermögen zwar partiell, sozio-emotionale Inhalte zu transportieren. Multimedialität erleichtert den Umgang mit räumlicher Information. Werden wenig reichhaltige Medien zur Bewältigung von gemeinsamen Aufgaben gewählt, kann mediale Depersonalisierung Statusmerkmale verbergen und vor Vorurteilen anderer Kommunikationsteilnehmer schützen. Asynchrone CvK gewährt Diskursteilnehmern Zeit zur Reflexion. Die Leichtigkeit elektronischer Speicherung fördert die Nachvollziehbarkeit und Möglichkeit zur Revision von Argumenten. Dennoch filtert CvK wesentliche Aspekte von Leiblichkeit aus. In ethisch-politischen Diskursen bedarf sie daher zumindest der Ergänzung durch ,korpo-reale“ Begegnung. ${ }^{2}$

2 Dem wurde bspw. im partizipatorischen Projekt „World Wide Views on Global Warming“ Rechnung getragen (siehe <http://www.wwviews.org/>. 


\section{E-Partizipation als „Diskursethik light“"}

Theorien der partizipatorischen Demokratie teilen mit jenen der deliberativen Demokratie das Ideal, Bürger auf breiter Basis in Prozesse argumentativ abwägender Beratschlagung einzubeziehen. Allerdings bestehen sie gegen die Gefahr von Elitenbildung und politischer Entfremdung auf einer „politics of amateurs“ (Barber 2003, 152), die sich (zumindest in Ergänzung zu den Institutionen des Repräsentativsystems) auch in Gremien bürgerlicher Selbstverwaltung durch die aktive Teilnahme an Entscheidungsprozessen realisiert. Projekte, die heute unter dem Titel der e-Participation firmieren, sind zwar diskursethisch unterfüttert, transportieren aber vielfach nicht mehr als eine „Diskursethik light“. Sie sind zumeist auf die Prämisse reduziert, dass die Diskursteilnehmer in ihren Artikulationen gleichberechtigt agieren. Der zentrale Aspekt der Teilhabe an Entscheidungsund Verfügungsgewalt bleibt ausgeblendet. Der Bescheidenheit solcher Praxis wird dadurch Vorschub geleistet, dass die Begriffe ,partizipatorische Demokratie“ und „Partizipation“ in der politischen Arena eine große Bandbreite unterschiedlicher Vorstellungen über die Rechte und Pflichten von Beteiligten bezeichnen.

So werden in der Fassung des gescheiterten Vertrags über eine „Verfassung für Europa“ von 2004 die Bestimmungen des Artikel I-47 noch mit der Überschrift „Grundsatz der partizipativen Demokratie“ versehen (die dann im nachfolgenden Vertrag von Lissabon 2007 nicht mehr aufscheint). Die Organe der Union werden dort sehr allgemein angehalten, einen „offenen, transparenten und regelmäßigen Dialog“ mit den Unionsbürgern zu ermöglichen und zu pflegen, sowie Anhörungen mit Betroffenen durchzuführen. Zudem sieht Artikel I-47 (4) die direktdemokratische Möglichkeit eines Volksbegehrens (im Text als „Bürgerinitiative“ bezeichnet) vor, das an die Europäische Kommission gerichtet ist, wobei die genauere Ausgestaltung des Instruments und der Bestimmungen, wie dann im Weiteren mit den Inhalten einer solchen Initiative umzugehen wäre, noch in Diskussion sind (siehe Amtsblatt der Europäischen Union 2004, C 310/34-C 310/35; Europäische Kommission 2010). Modelle mit differenzierten Abstufungen von Partizipation, die sich in einigen Varianten in Broschüren der öffentlichen Verwaltung finden lassen, stellen Skalen vor, die von der bloßen Weitergabe von Informationen bis zur aktiven Teilnahme an Entscheidungsprozessen reichen (vgl. etwa Bundeskanzleramt und BM für Land- und Forstwirtschaft, Umwelt und Wasserwirtschaft 2009, 24-25).

Die Vieldeutigkeit des Partizipationsbegriffs erlaubt es, sich einerseits auf unverbindliche und konventionelle Beteiligungsformen zurückzuziehen, andererseits aber den Elan der Volkssouveränität zu beschwören. Es ist wiederum die Literatur aus dem Umfeld der politischen Institutionen und der anwendungsorientierten Begleitforschung, die mit ihren Beschreibungen von e-Participation beizeiten eine Erwartungshaltung zugunsten ungezügelter sozialer Selbstorganisation nährt. Dabei ist ein sanfter Technikdeterminismus am Werk, der eine kausale Beziehung zwischen den funktionalen Möglichkeiten wechselseitiger Kommunikation durch den Gebrauch von IKT und egalitärer Diskursorganisation suggeriert: „In eParticipation processes ICTs empower humans, groups, and society, i.e. they provide individuals with capacities and resources for changing organizations and society according to their will, they provide groups and organizations with capacities and resources for changing society and better including individuals, and they provide society with capacities to better include groups and individuals (Fuchs et al. 2007, 6). Zugegeben: Das Zitat mag ein krasses Beispiel für jene technikaffinen Demokratieversprechen bieten, deren Charme sich aus einer Vermengung des Faktischen mit dem Möglichen und dem Erwünschten nährt, es ist aber mit seiner Ermächtigungsrhetorik nicht untypisch. Häufig anzutreffen im Diskurs über die elektronische Demokratie ist gerade die Spanne zwischen niederschwelliger Beteiligung und mobilisierender Rhetorik, in der e-Partizipation sich zum bloßen Instrument für die Erhöhung der Akzeptanz politischer Maßnahmen, als Mittel der strategischen Kommunikation im Sinne der ,public relations“ justieren lässt. 


\section{I Postdemokratie}

Abgesehen von den oben skizzierten theoretischen Schwierigkeiten, in der Praxis angemessene Diskursverfahren zu entwickeln, lässt sich die theoretisch überbordende, in der Umsetzung bescheidene, aber insgesamt unklare Anspruchshaltung, mit der e-Partizipation einhergeht, nur im Rahmen geänderter politischer Rahmenbedingungen verstehen: Anders als im Zeitalter der Massenmedien vor 40 Jahren stehen sich heute die Begriffe „Öffentlichkeit“ und „Gegenöffentlichkeit“ nicht mehr in einer einfachen Mechanik von ,hegemonial versus marginal“ gegenüber. Das Internet hat für eine Pluralisierung von Öffentlichkeiten (Münker 2009) gesorgt, in der Version des Web 2.0 mit Social Software das Paradox ,,persönlicher Öffentlichkeiten“ (Schmidt 2009) hervorgebracht. Selbstverständlich gibt es auch heute noch Bürgerinitiativen, die gegen die diskursive Hegemonie etablierter politischer Akteure ankämpfen, und gerade der aktuelle Konflikt um Informationsfreiheit im Zusammenhang mit den Veröffentlichungen durch WikiLeaks stellt eine vehemente Auseinandersetzung um das Prinzip Öffentlichkeit selbst dar. Allerdings wird heute Beteiligung nicht mehr allein von „,unten“ oder einem „Rand der Gesellschaft“, gefordert, sondern ist ein Thema, das vor allem von zwei politischen Akteuren vorangetrieben wird: von den NGOs, von denen viele im Rahmen neuer sozialer Steuerungsmechanismen an Einfluss gewonnen haben, und von der öffentlichen Verwaltung. Es würde den Rahmen dieses Beitrags sprengen, hier auf das Agieren der NGOs zwischen Gemeinwesenorientierung und Marktfähigkeit einzugehen und das zunehmende Outsourcing staatlicher Agenden in den Dritten Sektor zu beleuchten (vgl. dazu Brand 2004). Ich beschränke mich auf die Bemerkung, dass sich vormalige ,grass-roots“-Initiativen vielfach als kostengünstige öffentliche Dienstleister etabliert haben (bspw. im Bereich sozialer Leistungen, in der Bewältigung ökologischer Probleme oder in der Entwicklungszusammenarbeit) und im Vorfeld jener Administrationen agieren, die elektronische Partizipation ,top-down“ zu vermitteln versuchen.

e-Partizipation lässt sich als reaktives Instrument von Machteliten verstehen, um jene Legitimitätsprobleme einzudämmen, die in den 1990ern unter dem Titel „Krise der Repräsentation“ abgehandelt wurden und für die Colin Crouch (2008) später den populär gewordenen Begriff „Postdemokratie“ lieferte. Politikverdrossenheit gehört mit zu den Symptomen dieses postdemokratischen Zeitalters und genau die gelte es mit den neuen informationstechnischen Möglichkeiten wechselseitiger Kommunikation zu bekämpfen, denn, so die Europäische Kommission erstmals in einem White Paper 2001: ,[P] eople increasingly distrust institutions and politics or are simply not interested in them" (Commission of the European Communities 2001, 3). Die drohende Entkoppelung des Demos vom institutionalisierten politischen Geschehen ist bis heute das wesentliche Argument für eine Propagierung von e-Partizipation geblieben: „The challenge is to close a widening democratic gap between policymakers and citizens", heißt es demgemäß auch im Jahr 2010 programmatisch auf der Website des Klimawandel-Projekts „World Wide Views“, das sich dem Anliegen widmet, digitale Werkzeuge für eine weltweite Debatte zu nutzen.

\section{I.I Ökonomisierung und Legitimität}

Nach Crouch ist es die Ökonomisierung der Politik, die für diese Kluft zwischen dem Wahlvolk und seinen Repräsentanten verantwortlich ist und damit einer Stärkung autoritärer Tendenzen Vorschub leistet: „Die Globalisierung der wirtschaftlichen Eliten und die Fragmentierung der restlichen Bevölkerung tragen dazu bei, dass die sozialen Kräfte, denen es um einen Abbau ökonomischer und sozialer Ungleichheit geht, gegenüber jenen an Einfluss verlieren, die zu der hierarchischen Ordnung zurückkehren wollen, die wir aus vordemokratischen Zeiten kennen“ (2008, 34). Wenn dann nationalstaatliche Politik, auch angesichts ihrer Souveränitätsverluste im globalisierten Kapitalismus, in erster Linie von PR-Experten betrieben wird - ich verweise mit Thomas Meyer (2001) auf die These von einer „Kolonisierung der Politik durch die Medien“ - verliert sie ihre Gefolgschaft. 
e-Partizipation, die von formalen Verfahren der Entscheidungsfindung entkoppelt ist, läuft unter solchen Voraussetzungen aber paradoxerweise Gefahr, den Trend zur Entdemokratisierung zu verstärken statt ihm entgegenzuwirken. Für sie gilt, was Crouch (2008, 24 ff.) über die liberale Demokratie US-amerikanischen Zuschnitts behauptet: dass politische Beteiligung jenseits von Wahlen jene Personengruppen bevorzugt, auf Entscheidungen Einfluss zu nehmen, die über privilegierte Zugangsmöglichkeiten und finanzielle Ressourcen verfügen, wodurch der demokratische Grundsatz der formalen Gleichheit von Staatsbürgern untergraben wird. In diesem Sinn sollte man etwa für die starken Verzerrungen aufmerksam sein, wie sie in einer Untersuchung der EU-weiten Plattform „Your Voice in Europe“ konstatiert wurden: Die Inhaltsanalyse der von den Nutzern erstellten Beiträge zur Politikgestaltung in der Europäischen Union zeigte, dass die Hälfte aller Diskussionsbeiträge von nur $11 \%$ der Teilnehmer stammten. Die mit der Plattform verbundenen Online-Konsultationen für europäische Gesetzesvorhaben hinterliessen bei vielen Beteiligten das Gefühl, so sagten sie in Interviews, mit einer anonymen „Maschinerie“ zu kommunizieren, da sie kein inhaltliches Feedback bekamen (Winkler und Kozeluh 2006, 2-3).

Ökonomisierung der Politik ist aber nicht nur ein Makrophänomen. Ihre Effekte können auch auf der Ebene impliziter Diskursregeln gefunden werden, und das auch im Umfeld zivilgesellschaftlicher Selbstorganisation. 2006 untersuchten wir an den FH-Studiengängen Burgenland die OnlineDiskussionen und Entscheidungsstrukturen einer Gruppe österreichischer „Netizens“ (net + citizen), also einer elektronischen Bürgerinitiative. In der Gruppe hatte sich für das Agenda-Setting und für Entscheidungen ein Prinzip durchgesetzt, das Holtgrewe und Brand (2007) in einem anderen Zusammenhang, nämlich im Fall der Kooperation in Open-Source-Projekten als ,Norm der Verantwortlichkeit“ bezeichneten. Sie lautet schlicht: Entscheiden darf, wer zu einem bestimmten Anliegen oder Thema Arbeit investiert (ebenda, 37, 39). In der gemeinsamen Reflexion dieses Mechanismus vermerkten einige unserer Netizens, dass er nicht ihren eigenen Vorstellungen von demokratischer Teilhabe entspräche und mit verantwortlich für die mangelnde Offenheit der Gruppe gegenüber neuen Mitgliedern sein könnte. Andererseits fand aber auch das Argument, dass diese Norm der Verantwortlichkeit organisatorische Effizienz nach dem Vorbild privatwirtschaftlicher Unternehmen mit sich brächte, große Zustimmung (siehe Betz et al. 2006, 82-84, Betz 2008, 5).

Mit einem solchen Konnex von Mitsprache und Mitarbeit sei noch einmal auf den gesellschaftlichen Kontext verwiesen, in dem „Partizipation“ hilft, die Grenzen zwischen der Teilnahme an politischen Diskursen und der Leistungserbringung in ökonomischen Verwertungszusammenhängen zu verwischen. Mit der Erosion wohlfahrtstaatlicher Leistungen und der Deregulierung des Marktgeschehens setzen verstärkt Konzepte von „New Governance“ auf das Selbstmanagement und die Selbstversorgung der Bürger im Hinblick auf die Bewältigung individueller Risiken und die Bereitstellung vormals öffentlicher Güter (siehe Rose 1996). Unter solchen Voraussetzungen wird dann auch (elektronische) Partizipation als funktionale Anforderung verstanden, die den Rückzug staatlicher Versorgung durch ,active citizenship“, das heißt unter anderem durch die Mobilisierung sozialen Kapitals, von ehrenamtlichen Tätigkeiten und gemeinschaftlicher Kapazitäten der Problemlösung kompensiert (Smith und Dalakiouridou 2009, 4, 6). Auf betrieblicher Ebene wiederum sind es computerunterstützte postfordistische Konzepte der Arbeitsorganisation, die unter Etiketten wie „eCollaboration“ oder „Enterprise 2.0“ firmieren und zugunsten erhöhter Produktivität die Partizipation der Arbeitnehmer in der unternehmensinternen Kommunikation (und in der Kommunikation mit Kunden) als persönliches, aktives Engagement abseits von Hierarchien und Weisungsgebundenheit zu mobilisieren versuchen. Wie in politischen Fragen (Kuhlen 2006), so werden auch im Unternehmensbereich die gesteigerten Möglichkeiten zur ortsunabhängigen manyto-many-Kommunikation durch Social Software als wesentliche Voraussetzung intensivierter Beteiligung gesehen (beispielhaft Kock 2005, McAfee 2006, Döbler 2008). 


\section{Schlussfolgerungen}

Crouch $(2008,20)$ nennt zwei mögliche Wege ,zur Belebung der Demokratie“. Entweder sind das „Krisen und Veränderungen, die ein erneutes politisches Engagement hervorrufen“, oder es ist, was er im Rahmen westlicher Demokratien für wahrscheinlicher hält, „die Entstehung neuer kollektiver Identitäten, die die Form der Partizipation an Debatten und Entscheidungen verändern.“

Zweifel scheinen berechtigt, dass technisch-ökologisch-soziale Desaster wie der Klimawandel allein aufgrund des Problemdrucks sozialen Wandel zugunsten einer gesteigerten kollektiven Entscheidungsrationalität bewirken. Schon anlässlich des Berliner Gentechnik-Diskurses vor beinahe zwei Jahrzehnten wurde festgestellt, dass ein „Einigungsdruck aus der ,Natur der Sache”“, allein nicht ausreichte, sondern erst durch das diskursive Setting, das die Veranstalter kreierten, „mobilisiert werden" musste (Jansen 2000, 185).

Wenn es Projekten politischer Partizipation, die (auch, aber nicht ausschließlich) elektronische Räume nutzen, gelingt, Machtasymmetrien vorzubeugen, also die formale staats-, besser weltbürgerliche Gleichheit der Teilnehmer sicherzustellen, können sie möglicherweise zur Entstehung oder Sichtbarmachung neuer kollektiver Identitäten beitragen, die wiederum Einfluss auf die Form des öffentlichen Diskurses nehmen. Projekte der e-Partizipation müssten dazu allerdings ein Selbstverständnis beisteuern, das auf eine Trennschärfe zwischen politischer Beteiligung und Partizipation im Sinn ökonomischer Verwertungslogik Wert legt.

Eine Ethik des Diskurses in Anspruch zu nehmen, bedeutet, sich in der praktischen Organisation von Deliberation mit hohen Forderungen auseinandersetzen: Im Zusammenhang mit e-Partizipation ist hier auf die Notwendigkeit zu verweisen, in der Strukturierung von Debatten der Begründung von Argumenten besonderes Augenmerk zu schenken. Für die Attraktivität von Beteiligungsprozessen ist zudem, so habe ich in diesem Beitrag argumentiert, eine enge Verbindung mit Modi der Entscheidungsfindung bei gleichzeitiger Verabschiedung von starker Konsensorientierung wichtig. Das kann im Fall überschaubarer Kommunikationsgemeinschaften eine Kopplung der Debatte mit verfahrensregulierter Entscheidung bedeuten. Im Fall der Konsultation in einer breiten Öffentlichkeit wäre für gesteigerte Transparenz zu sorgen, wenn Vorschläge an politische Repräsentanten oder Experten zur Entscheidungsfindung delegiert werden.

E-Partizipation kann im Web 2.0 aus einer multimedialen Palette von Kommunikationsmöglichkeiten wählen, und dabei immer wieder die Passung zwischen themenspezifischen Kommunikationsanforderungen und Medieneigenschaften erproben. Aus leibphilosophischer Perspektive lässt sich dann die Forderung erheben, sich im Diskurs nicht allein auf die vielfältigen Möglichkeiten der CvK zu beschränken, sondern temporär auch die korpo-reale Präsenz in die Medienwahl einzubeziehen. Die mediale Reichhaltigkeit des Leibs ist von Zeit zu Zeit unumgänglich, damit sich Diskursteilnehmer als Personen mit ihren sozialstrukturellen und identitätspolitischen Unterschieden wahrnehmen, adressieren und respektieren können. 


\section{Literatur}

Alle im Quellenverzeichnis angeführten Weblinks wurden zuletzt Ende November 2010 aufgerufen.

Apel, K.-O., 1999, Transformation der Philosophie, Bd. 2, Das Apriori der

Kommunikationsgemeinschaft, 6. Auflage, Frankfurt/Main: Suhrkamp (orig. 1976).

Amtsblatt der Europäischen Union, 2004, Vertrag über eine Verfassung für Europa, 2004/C 310/01, 47. Jg., 16. Dezember 2004,

<http://eur-lex.europa.eu/LexUriServ/LexUriServ.do?uri=OJ:C:2004:3 1 0:00 I 1:0040:DE:PDF>.

Barber, B., 2003, Strong Democracy. Participatory Politics for a New Age, Berkeley und Los Angeles: University of California Press (orig. 1984).

Beck, U., 1986, Risikogesellschaft. Auf dem Weg in eine andere Moderne, Frankfurt/Main: Suhrkamp.

Betz, F., 2008, eGovernment und eGovernmentality in Österreich, in: Zimmer, B. und Koubek, A. (Hg): Erstes Forschungsforum der österreichischen Fachhochschulen, Tagungsband, 11./12. April 2007, Fachhochschule Salzburg, Campus Urstein. München: Verlag Meidenbauer, 109-114.

Betz, F. et al., 2006, How Democratic is eGovernment? Public Knowledge Management and Governmentality in Europe, Endbericht an das BM für Bildung, Wissenschaft und Kultur, Eisenstadt: FH-Studiengänge Burgenland.

Böhme, G., 2003, Leibsein als Aufgabe. Leibphilosophie in pragmatischer Hinsicht, Zug: Die Graue Edition.

Brand, U., 2004, Governance, in: Bröckling, U. et al. (Hg.): Glossar der Gegenwart, Frankfurt/Main: Suhrkamp, 111-117.

Bundeskanzleramt und BM für Land- und Forstwirtschaft, Umwelt und Wasserwirtschaft, 2009, Standards der Öffentlichkeitsbeteiligung, Empfehlungen für die gute Praxis, $<$ http://www.partizipation.at/fileadmin/media_data/Downloads/Standards_OeB/ standards_der_oeffentlichkeitsbeteiligung_2008_druck.pdf>.

Candiwi, 2010, <http://www.metagovernment.org/wiki/Candiwi>.

Commission of the European Communities, 2001, European Governance. A White Paper, COM (2001) 428, Brussels, 25. 7. 2001, <http://ec.europa.eu/governance/white_paper/en.pdf>.

Crouch, C., 2008, Postdemokratie, Frankfurt/Main: Suhrkamp (orig. 2003).

Derrida, J., 2003, Schurken. Zwei Essays über die Vernunft, übers. von Horst Brühmann. Frankfurt/Main: Suhrkamp.

Döbler, T., 2008, Zum Einsatz von Social Software in Unternehmen, in: Stegbauer, C. und Jäckel, M. (Hg.): Social Software, Formen der Kooperation in computerbasierten Netzwerken, Wiesbaden: Verlag für Sozialwissenschaften, 119-136.

Europäische Kommission, 2010, Vorschlag für eine Verordnung des Europäischen Parlaments und des Rates über die Bürgerinitiative, $\operatorname{KOM(2010)~} 119$ endgültig, Brüssel, 31. 3. 2010, $<$ http://ec.europa.eu/dgs/secretariat_general/citizens_initiative/docs/com_2010_II9_de.pdf>. 
Fuchs, C. et al., 2006, 'Broadening eParticipation: Rethinking ICTs and Participation'. Association of Internet Researchers Conference: Internet Research 7.0, 27.-30. September, Brisbane, ICT\&S Center Research Paper Series No. 2, Salzburg: ICT\&S Center, $<$ http://www.gov2u.org/publications/Broadening_eParticipation.pdf>.

Groote, M., 2009, Eröffnungsrede zum „eParticipation Day 2009” am 04.03.2009 in der Europäischen Kommission. E-Partizipation - Chancen und Herausforderungen für die parlamentarische Arbeit im Europäischen Parlament,

$<$ http://ec.europa.eu/information_society/events/eparticipation/2009/conference/presentati ons/speech_groote_04032009.pdf>.

Habermas, J., 1983, Moralbewußtsein und kommunikatives Handeln, Frankfurt/Main: Suhrkamp.

Habermas, J., 1991, Erläuterungen zur Diskursethik, Frankfurt/Main: Suhrkamp.

Habermas, J., 1994, Faktizität und Geltung. Beiträge zur Diskurstheorie des Rechts und des demokratischen Rechtsstaats, 4. Auflage, Frankfurt/Main: Suhrkamp.

Holtgrewe, U. und Brand, A. 2007, Die Projektpolis bei der Arbeit. Open-Source-SoftwareEntwicklung und der ,neue Geist des Kapitalismus“, Österreichische Zeitschrift für Soziologie 32(3), 25-45.

Jansen, D., 2000, Gesellschaftliche Selbstorganisation durch Technikdiskurse? in: Werle, R. und Schimank, U. (Hg.): Gesellschaftliche Komplexität und kollektive Handlungsfähigkeit. Frankfurt/Main: Campus, 183-207.

Kock, N., 2005, What is E-Collaboration? Editorial Essay, International Journal of e-Collaboration 1(1), i-vii.

Kuhlen, R., 2006, Potenziale einer politischen Kollaborationskultur, <http://www.kuhlen.name/MATERIALIEN/Publikationen2007/ potenziale-kollaborationskultur-RK-in-Leggewie2007.pdf>.

Laclau, E. und Mouffé, C., 1991, Hegemonie und radikale Demokratie. Zur Dekonstruktion des Marxismus. Wien: Passagen Verlag (engl. 1985).

List, E., 2009, Ethik des Lebendigen. Weilerswist: Velbrück Wissenschaft.

Lösch, B., 2005, Deliberative Politik. Moderne Konzeptionen von Öffentlichkeit, Demokratie und politischer Partizipation, Münster: Westfälisches Dampfboot.

Macintosh, A., 2004, Characterizing E-Participation in Policy-Making, in: Proceedings of the $37^{\text {th }}$ Hawaii International Conference on System Sciences - 2004, <http://citeseerx.ist.psu.edu>.

McAfee, A. P., 2006, Enterprise 2.0: The Dawn of Emergent Collaboration, MIT Sloan Management Review 47 (3), 21-38.

Metagovernment, 2010, <http://www.metagovernment.org/wiki/Collaborative_governance>.

Meyer, T., 2001, Mediokratie. Die Kolonisierung der Politik durch die Medien. Frankfurt/Main: Suhrkamp.

Mouffé, C., 2000, Deliberative Democracy or Agonistic Pluralism, Political Sciences Series Vol. 72, Wien: Institut für Höhere Studien (IHS).

Münker, S., 2009, Emergenz digitaler Öffentlichkeiten. Die Sozialen Medien im Web 2.0. Frankfurt/Main: Suhrkamp.

Rose, N., 1996, The death of the social? Re-figuring the territory of government, Economy and Society 25(3), 327-356. 
Schedler, K., Summermatter, L. und Schmidt, B., 2003, Electronic government einführen und entwickeln - von der Idee zur Praxis. Bern: Haupt.

Schmidt, J. (2009). Das neue Netz. Merkmale, Praktiken und Folgen des Web 2.0. Konstanz: UVK.

Smith, S. und Dalakiouridou, E., 2009, Contextualising Public (e)Participation in the Governance of the European Union, European Journal of ePractice 3(7), 4-14,

<http://www.epractice.eu/files/ePractice-Journal-Volume-7.pdf>.

Stickler, T., Prutsch, A. und Balas, M., 2010, Klimawandelanpassung in Österreich. Ergebnisse der Internet-Befragung im Rahmen des Beteiligungsprozesses zur Begleitung und Unterstützung der Erstellung der österreichischen Strategie zur Klimawandelanpassung, Wien: Umweltbundesamt $\mathrm{GmbH}$,

<http://www.ubavie.gv.at/fileadmin/site/publikationen/REP0266.pdf>.

Wetter-Klimawandel.de, 2010, <http://www.wetter-klimawandel.de/auswirkungen-klima.php>.

Wilhelm, A. G., 2000, Democracy in the Digital Age. Challenges to Political Life in Cyberspace, New York und London: Routledge.

Winkler, R. und Kozeluh, U., 2006, Europeans Have a Say: Online Debates and Consultations in the EU, Forschungsberichte 21, Wien: Bundesministerium für Bildung, Wissenschaft und Kultur, Abteilung für Gesellschaftswissenschaften.

Zizek, S., 1991, Liebe Dein Symptom wie Dich selbst! Jaques Lacans Psychoanalyse und die Medien. Berlin: Merve.

Zizek, S., 1997, Mehr-Genießen, 2. Auflage. Wien: Turia + Kant. 


\section{Bisher erschienene manu:scripte}

ITA-0I-0I Gunther Tichy, Walter Peissl (12/2001): Beeinträchtigung der Privatsphäre in der Informationsgesellschaft.

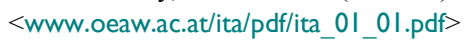

ITA-0I-02 Georg Aichholzer(12/2001): Delphi Austria: An Example of Tailoring Foresight to the Needs of a Small Country.

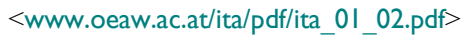

ITA-0I-03 Helge Torgersen, Jürgen Hampel (12/2001): The Gate-Resonance Model: The Interface of Policy, Media and the Public in Technology Conflicts. <www.oeaw.ac.at/ita/pdf/ita_0I_03.pdf>

ITA-02-0I Georg Aichholzer (01/2002): Das ExpertInnen-Delphi: Methodische Grundlagen und Anwendungsfeld „Technology Foresight“. <www.oeaw.ac.at/ita/pdf/ita_02_01.pdf>

ITA-02-02 Walter Peissl (01/2002): Surveillance and Security - A Dodgy Relationship. $<w w w$. oeaw.ac.at/ita/pdf/ita_02_02.pdf>

ITA-02-03 Gunther Tichy (02/2002): Informationsgesellschaft und flexiblere Arbeitsmärkte. $<w w w . o e a w . a c . a t / i t a / p d f / i t a \_02 \_03 . p d f>$

ITA-02-04 Andreas Diekmann (06/2002): Diagnose von Fehlerquellen und methodische Qualität in der sozialwissenschaftlichen Forschung. <www.oeaw.ac.at/ita/pdf/ita_02_04.pdf>

ITA-02-05 Gunther Tichy (10/2002): Over-optimism Among Experts in Assessment and Foresight. $<$ www.oeaw.ac.at/ita/pdf/ita_02 05.pdf>

ITA-02-06 Hilmar Westholm (12/2002): Mit eDemocracy zu deliberativer Politik? Zur Praxis und Anschlussfähigkeit eines neuen Mediums. <www.oeaw.ac.at/ita/pdf/ita_02_06.pdf>

ITA-03-0I Jörg Flecker und Sabine Kirschenhofer (01/2003): IT verleiht Flügel? Aktuelle Tendenzen der räumlichen Verlagerung von Arbeit. <www.oeaw.ac.at/ita/pdf/ita_03_0l.pdf>

ITA-03-02 Gunther Tichy (11/2003): Die Risikogesellschaft - Ein vernachlässigtes Konzept in der europäischen Stagnationsdiskussion. < www.oeaw.ac.at/ita/pdf/ita_03_02.pdf>

ITA-03-03 Michael Nentwich (11/2003): Neue Kommunikationstechnologien und Wissenschaft - Veränderungspotentiale und Handlungsoptionen auf dem Weg zur Cyber-Wissenschaft. <www.oeaw.ac.at/ita/pdf/ita_03_03.pdf>

ITA-04-0I Gerd Schienstock (1/2004): Finnland auf dem Weg zur Wissensökonomie - Von Pfadabhängigkeit zu Pfadentwicklung. <www.oeaw.ac.at/ita/pdf/ita_04_0I.pdf>

ITA-04-02 Gunther Tichy (6/2004): Technikfolgen-Abschätzung: Entscheidungshilfe in einer komplexen Welt. $<$ www.oeaw.ac.at/ita/pdf/ita 04 02.pdf>

ITA-04-03 Johannes M. Bauer (11/2004): Governing the Networks of the Information Society - Prospects and limits of policy in a complex technical system. <www.oeaw.ac.at/ita/pdf/ita_04_03.pdf>

ITA-04-04 Ronald Leenes (12/2004): Local e-Government in the Netherlands: From Ambitious Policy Goals to Harsh Reality. <www.oeaw.ac.at/ita/pdf/ita_04_04.pdf>

ITA-05-0I Andreas Krisch (01/2005): Die Veröffentlichung des Privaten - Mit intelligenten Etiketten vom grundsätzlichen Schutz der Privatsphäre zum Selbstschutz-Prinzip. <www.oeaw.ac.at/ita/pdf/ita_05_0I.pdf >

ITA-05-02 Petra Grabner (12/2005): Ein Subsidiaritätstest - Die Errichtung gentechnikfreier Regionen in Österreich zwischen Anspruch und Wirklichkeit. <www.oeaw.ac.at/ita/pdf/ita_05_02.pdf>

ITA-05-03 Eva Buchinger (12/2005): Innovationspolitik aus systemtheoretischer Sicht - Ein zyklisches Modell der politischen Steuerung technologischer Innovation. <www.oeaw.ac.at/ita/pdf/ita_05_03.pdf $>$

ITA-06-0I Michael Latzer (06/2006): Medien- und Telekommunikationspolitik: Unordnung durch Konvergenz Ordnung durch Mediamatikpolitik. <epub.oeaw.ac.at/ita/ita-manuscript/ita_06_0I.pdf>

ITA-06-02 Natascha Just, Michael Latzer, Florian Saurwein (09/2006): Communications Governance: Entscheidungshilfe für die Wahl des Regulierungsarrangements am Beispiel Spam. <epub.oeaw.ac.at/ita/ita-manuscript/ita_06_02.pdf>

ITA-06-03 Veronika Gaube, Helmut Haberl (10/2006): Sozial-ökologische Konzepte, Modelle und Indikatoren nachhaltiger Entwicklung: Trends im Ressourcenverbrauch in Österreich. <epub.oeaw.ac.at/ita/ita-manuscript/ita_06_03.pdf>

ITA-06-04 Maximilian Fochler, Annina Müller (11/2006): Vom Defizit zum Dialog? Zum Verhältnis von Wissenschaft und Öffentlichkeit in der europäischen und österreichischen Forschungspolitik. <epub.oeaw.ac.at/ita/ita-manuscript/ita_06_04.pdf>

ITA-06-05 Holger Floeting (11/2006): Sicherheitstechnologien und neue urbane Sicherheitsregimes. <epub.oeaw.ac.at/ita/ita-manuscript/ita_06_05.pdf>

ITA-06-06 Armin Spök (12/2006): From Farming to „Pharming”- Risks and Policy Challenges of Third Generation GM Crops. <epub.oeaw.ac.at/ita/ita-manuscript/ita_06_06.pdf>

ITA-07-0I Volker Stelzer, Christine Rösch, Konrad Raab (3/2007): Ein integratives Konzept zur Messung von Nachhaltigkeit - das Beispiel Energiegewinnung aus Grünland. $<$ epub.oeaw.ac.at/ita/ita-manuscript/ita_07_01.pdf> 
ITA-07-02 Elisabeth Katzlinger (3/2007): Big Brother beim Lernen: Privatsphäre und Datenschutz in Lernplattformen. $<$ epub.oeaw.ac.at/ita/ita-manuscript/ita_07_02.pdf>

ITA-07-03 Astrid Engel, Martina Erlemann (4/2007): Kartierte Risikokonflikte als Instrument reflexiver Wissenspolitik. <epub.oeaw.ac.at/ita/ita-manuscript/ita_07_03.pdf>

ITA-07-04 Peter Parycek (5/2007): Gläserne Bürger - transparenter Staat? Risiken und Reformpotenziale des öffentlichen Sektors in der Wissensgesellschaft. <epub.oeaw.ac.at/ita/ita-manuscript/ita_07_04.pdf>

ITA-07-05 Helge Torgersen (7/2007): Sicherheitsansprüche an neue Technologien - das Beispiel Nanotechnologie. <epub.oeaw.ac.at/ita/ita-manuscript/ita_07_05.pdf>

ITA-07-06 Karen Kastenhofer (9/2007): Zwischen „schwacher“ und ,starker“ Interdisziplinarität. Die Notwendigkeit der Balance epistemischer Kulturen in der Sicherheitsforschung zu neuen Technologien.

<epub.oeaw.ac.at/ita/ita-manuscript/ita_07_06.pdf>

ITA-07-07 Ralf Lindner, Michael Friedewald (9/2007): Gesellschaftliche Herausforderungen durch ,intelligente Umgebungen. Dunkle Szenarien als TA-Werkzeug. <epub.oeaw.ac.at/ita/ita-manuscript/ita_07_07.pdf>

ITA-07-08 Alfons Bora (11/2007): Die disziplinären Grundlagen der Wissenschaft. <epub.oeaw.ac.at/ita/ita-manuscript/ita_07_08.pdf>

ITA-08-0I Alexander Degelsegger (5/2008): „Frames“ in sozialwissenschaftlichen Theorieansätzen. Ein Vergleich aus der Perspektive der Technikforschung. <epub.oeaw.ac.at/ita/ita-manuscript/ita_08_0I.pdf>

ITA-08-02 Jens Hoff (11/2008): Can The Internet Swing The Vote? Results from a study of the 2007 Danish parliamentary election. <epub.oeaw.ac.at/ita/ita-manuscript/ita_08_02.pdf>

ITA-09-0I Georg Aichholzer, Doris Allhutter (2/2009): e-Participation in Austria: Trends and Public Policies. <epub.oeaw.ac.at/ita/ita-manuscript/ita_09_0I.pdf>

ITA-09-02 Michael Nentwich (11/2009): Cyberscience 2.0 oder 1.2? Das Web 2.0 und die Wissenschaft. <epub.oeaw.ac.at/ita/ita-manuscript/ita_09_02.pdf>

ITA-09-03 Hilmar Westholm (12/2009): Wandel der Formen politischer Partizipation und der Beitrag des Internet. Schlussfolgerungen aus Bevölkerungsbefragungen in Deutschland. <epub.oeaw.ac.at/ita/ita-manuscript/ita_09_03.pdf>

ITA-10-0I Iris Eisenberger (12/2010): Kleine Teile, große Wirkung? Nanotechnologieregulierung in der Europäischen Union. <epub.oeaw.ac.at/ita/ita-manuscript/ita_I0_0I.pdf>

ITA-10-02 Alexander Degelsegger and Helge Torgersen (12/2010): Instructions for being unhappy with PTA. The impact on PTA of Austrian technology policy experts' conceptualisation of the public. <epub.oeaw.ac.at/ita/ita-manuscript/ita_10_02.pdf>

ITA-10-03 Ernest Braun (12/2010): The Changing Role of Technology in Society. <epub.oeaw.ac.at/ita/ita-manuscript/ita_10_03.pdf>

ITA-10-04 Fritz Betz (12/2010): E-Partizipation und die Grenzen der Diskursethik. <epub.oeaw.ac.at/ita/ita-manuscript/ita_10_04.pdf> 